\title{
RAIRO
}

ANALYSE NUMÉRIQUE

\author{
JIM JR. DOUGLAS
}

TODD DUPONT

HENRY H. JR. RACHFORD

MARY F. WHEELER

\section{Local $H^{-1}$ Galerkin and adjoint local $H^{-1}$ Galerkin procedures for elliptic equations}

RAIRO - Analyse numérique, tome 11, no 1 (1977), p. 3-12.

<http://www.numdam.org/item?id=M2AN_1977_11_1_3_0>

(C) AFCET, 1977, tous droits réservés.

L'accès aux archives de la revue «RAIRO - Analyse numérique » implique l'accord avec les conditions générales d'utilisation (http://www.numdam.org/ legal.php). Toute utilisation commerciale ou impression systématique est constitutive d'une infraction pénale. Toute copie ou impression de ce fichier doit contenir la présente mention de copyright.

\section{Numdam}




\title{
LOCAL $H^{-1}$ GALERKIN \\ AND ADJOINT LOCAL $H^{-1}$ GALERKIN \\ PROCEDURES FOR ELLIPTIC EQUATIONS (*)
}

\author{
by Jim Douglas, Jr. $\left({ }^{1}\right)$, \\ Todd Dupont $\left({ }^{1}\right)$, Henry H. RaChFORD, Jr. $\left({ }^{2}\right)$ \\ and Mary F. WhEELER $\left({ }^{2}\right)$
}

\begin{abstract}
Two essentially dual, finite element methods for approximating the solution of the boundary value problem $L u=\nabla \cdot(a \nabla u)+b \cdot \nabla u+c u=f$ on $\Omega$, a rectangle, with $u=0$ on $\partial \Omega$ are shown to give optimal order convergence. The local $H^{-1}$ method is based on the inner product $\left(u, L^{*} v\right)$ and the adjoint method on $(L u, v)$. Discontinuous spaces can be employed for the approximate solution in the local $\mathrm{H}^{-1}$ procedure and for the test space in the adjoint method.
\end{abstract}

\section{INTRODUCTION}

Consider the elliptic boundary value boundary problem

$$
\left.\begin{array}{c}
(L u)(p)=\nabla \cdot(a(p) \nabla u)+b(p) \cdot \nabla u+c(p) u=f(p), \quad p \in \Omega, \\
u(p)=0, \quad p \in \partial \Omega,
\end{array}\right\}
$$

where $\Omega$ is the square $I \times I$ and $I=(0,1)$. We assume that $a,(\nabla a)_{i}, b_{i}$, $c \in C^{1}(\bar{\Omega})$, that $f \in L_{2}(\Omega)$, and that $0<a_{0} \leqq a(p) \leqq a_{1}, p \in \bar{\Omega}$, where $a_{0}$ and $a_{1}$ are constants. We further assume that, given $g \in L_{2}(\Omega)$, there exists a unique function $\varphi \in H^{2}(\Omega)$ satisfying $L \varphi=g$ in $\Omega$ and $\varphi=0$ on $\partial \Omega$.

We shall use the following notation. Let $\delta: 0=x_{0}<x_{1}<\ldots<x_{\mathrm{N}}=1$ be a partition of $[0,1]$. Set $I_{j}=\left(x_{j-1}, x_{j}\right), h_{j}=x_{j}-x_{j-1}$, and $h=\max h_{j}$.

For $E \subset I$ let $P_{r}(E)$ denote the functions defined on $I$ whose restrictions to $E$ coincide with polynomials of degree at most $r$. Let

$$
\mathscr{M}(-1, r, \dot{0})=\bigcap_{j=1}^{\mathrm{N}} P_{r}\left(I_{j}\right)
$$

and, for $k$ a non-negative integer,

$$
\begin{gathered}
\mathscr{M}(k, r, \delta)=\mathscr{M}(-1, r, \delta) \cap C^{k}(I), \\
\mathscr{M}^{0}(k, r, \delta)=\mathscr{M}(k, r, \delta) \cap\{v \mid v(0)=v(1)=0\}, \\
\tilde{\mathscr{U}}(k-1, r-1, \delta)=\left\{v^{\prime}: v \in \mathscr{M}^{0}(k, r, \delta)\right\} .
\end{gathered}
$$

(*) Reçu août 1975.

(1) University of Chicago, Chicago, Illinois 60637, U.S.A.

$\left.{ }^{2}\right)$ Rice University, Houston, Texas 77001 , U.S.A.

R.A.I.R.O. Analyse numérique/Numerical Analysis, vol. 11, n 1, 1977 
We assume that $\delta$ is quasi-uniform and that $r \geqq 1$. For brevity, we set

and

$$
\begin{gathered}
\mathscr{N}=\mathscr{M}^{0}(k+2, r+2, \delta) \otimes \mathscr{M}^{0}(k+2, r+2, \delta), \\
\mathscr{Q}=\tilde{\mathscr{M}}(k+1, r+1, \delta) \otimes \tilde{\mathscr{M}}(k+1, r+1, \delta),
\end{gathered}
$$

$$
\mathscr{M}=\mathscr{M}(k, r, \delta) \otimes \mathscr{M}(k, r, \delta) .
$$

Note that $\mathscr{Q}$ and $\mathscr{M}$ are the images of $\mathcal{N}$ under the maps given by $\partial^{2} / \partial x \partial y$ and $\partial^{4} / \partial x^{2} \partial y^{2}$, respectively.

The local $H^{-1}$ Galerkin approximation is defined as the solution $U \in \mathscr{M}$ of the equations

$$
\left(U, L^{*} \varphi\right)=(f, \varphi), \quad \varphi \in \mathcal{N},
$$

where the inner product is the standard $L_{2}(\Omega)$ one. The adjoint local $H^{-1}$ Galerkin approximation is given by $W \in \mathscr{N}$ satisfying

$$
(L W, \varphi)=(f, \varphi), \quad \varphi \in \mathscr{M} .
$$

We first show that there exists a unique $U$ and a unique $W$ satisfying (2) and (3), respectively, for $L=\Delta$. Optimal $L_{2}$ error estimates are also obtained for the operator $\Delta$. We then generalize our results to obtain optimal $L_{2}$ results for operators of the form given in (1).

Let $H^{k}(\Omega)$ be the Sobolev space of functions having $L_{2}(\Omega)$-derivatives through order $k$. Denote the usual norm on $H^{s}(\Omega)$ by $\|\cdot\|_{s}$; for $s=0$ the subscript will be omitted. We also use the norm

$$
\|w\|_{-1}=\sup _{z \varepsilon H^{1}(\Omega)} \frac{(w, z)}{\|z\|_{1}} .
$$

If the reader wishes to use any of the results derived below for non-integral indices; then standard interpolation theory [3] should be applied.

\section{ERROR ESTIMATES FOR $L=\Delta$}

First note that, since $\operatorname{dim} \mathscr{M}=\operatorname{dim} \mathscr{N}$, uniqueness implies existence.

Lemma 1 : Suppose that $V \in \mathscr{M}$ satisfies

Then, $V \equiv 0$.

$$
(V, \Delta \varphi)=0, \quad \varphi \in \mathscr{N} .
$$

Proof: Note that there exists a unique $Q \in \mathscr{N}$ such that $Q_{x x y y}=V$. Integrating by parts, we have

$$
\left(\nabla Q_{x y}, \nabla w\right)=0, \quad w \in \mathscr{Q} .
$$

Since $Q_{x y} \in \mathcal{Q}$, we note that $Q_{x x y}=0$ and $Q_{y y x}=0$. Thus, $V=0$.

Since the matrix arising in (3) is the adjoint of that of (2), there exists a unique $W$ satisfying (3) for $L=\Delta$. 
We now derive $L_{2}$ and negative norm error estimates for $U-u$ when $L=\Delta$. Let $Z \in \mathscr{N}$ satisfy $Z_{x x y y}=U$. Also let $z_{x x y y}=u$ in $\Omega$ and $z=0$ on $\partial \Omega$. We observe from (1) and (2) with $\xi=Z-z$ that

$$
\left(\nabla \xi_{x y}, \nabla w\right)=0, \quad w \in \mathscr{Q} .
$$

THEOREM 1 : Let $z$ and $Z$ be as defined above, and let $z_{x y} \in H^{s}(\Omega)$ for some $s$ such that $1 \leqq s \leqq r+2$. Then,

$$
\left\|(z-Z)_{x y}\right\|+h\left\|(z-Z)_{x y}\right\|_{1} \leqq C\left\|z_{x y}\right\|_{s} h^{s} .
$$

Proof : It follows from (4) that

$$
\left\|\nabla \xi_{x y}\right\|=\inf _{x \in \mathcal{Q}}\left\|\nabla\left(z_{x y}-\chi\right)\right\| .
$$

Let $T: H^{1}(I) \rightarrow \mathscr{M}(k+1, r+1, \delta)$ be determined by the relations

$$
\int_{0}^{1}(g-T g)^{\prime} v d x=\int_{0}^{1}(g-T g) d x=0, \quad v \in \mathscr{M}(k, r, \delta) .
$$

It is easy to see that $(g-T g)(0)=(g-T g)(1)=0$, by taking $v=x$ or $1-x$. Since $(T g)^{\prime}$ is the $L_{2}(I)$-projection of $g^{\prime}$ into $\mathscr{M}(k, r, \delta)$,

Let

$$
\left\|(g-T g)^{\prime}\right\|_{L_{2}(I)} \leqq C\left\|g^{(s)}\right\|_{L_{2}(I)} h^{s-1}, \quad 1 \leqq s \leqq r+2 .
$$

$$
\begin{gathered}
-\varphi^{\prime \prime}=\zeta=g-T g, \quad x \in I, \\
\varphi^{\prime}(0)=\varphi^{\prime}(1)=0, \\
\int_{0}^{1} \varphi d x=0 .
\end{gathered}
$$

Then for $v \in \mathscr{M}(k, r, \delta)$ appropriately chosen

and

$$
\|\zeta\|^{2}=\left(\zeta^{\prime}, \varphi^{\prime}-v\right) \leqq C\left\|\zeta^{\prime}\right\|_{L_{2}(I)}\|\zeta\|_{L_{2}(I)} h,
$$

$$
\|g-T g\|_{L_{2}(I)} \leqq C\left\|g^{(s)}\right\|_{L_{2}(I)} h^{(s)}, \quad 1 \leqq s \leqq r+2 .
$$

Consider $(T \otimes T) z_{x y} \in \mathscr{M}(k+1, r+1, \delta) \otimes \mathscr{M}(k+1, r+1, \delta)$. It is easy to see that $(T \otimes T) z_{x y} \in \mathscr{Q}$ and that

$$
\left\|z_{x y}-(T \otimes T) z_{x y}\right\|_{q} \leqq C\left\|z_{x y}\right\|_{s} h^{s-q}, \quad 2 \leqq s \leqq r+2, \quad 0 \leqq q \leqq 1,
$$

since $T \otimes T-I \otimes I=(T-I) \otimes I+I \otimes(T-I)+(T-I) \otimes(T-I)$. Thus, from (5) and (6),

The inequality

$$
\left\|\nabla \xi_{x y}\right\| \leqq C\left\|z_{x y}\right\|_{s} h^{s-1}, \quad 2 \leqq s \leqq r+2 .
$$

$$
\left\|\nabla \xi_{x y}\right\| \leqq C\left\|\nabla z_{x y}\right\| \leqq C\left\|z_{x y}\right\|_{1}
$$

vol. $11, \mathrm{n}^{\circ} 1,1977$ 
is obvious, and the desired result follows:

$$
\left\|\nabla \xi_{x y}\right\| \leqq C\left\|z_{x y}\right\|_{s} h^{s-1}, \quad 1 \leqq s \leqq r+2
$$

Since $\xi_{x y}$ has average value zero,

$$
\left\|\xi_{x y}\right\|_{1} \leqq C\left\|z_{x y}\right\|_{s} h^{s-1}, \quad 1 \leqq s \leqq r+2 .
$$

To obtain the $L_{2}(\Omega)$ estimate, first let

$$
\begin{aligned}
-\Delta \varphi & =\xi_{x y}, & (x, y) \in \Omega, \\
\frac{\partial \varphi}{\partial n} & =0, & (x, y) \in \partial \Omega .
\end{aligned}
$$

Since $\left(\xi_{x y}, 1\right)=0$, there exists $\varphi$ such that $(\varphi, 1)=0$ and $\|\varphi\|_{2} \leqq C\left\|\xi_{x y}\right\|$. Then,

and

$$
\begin{aligned}
& \left\|\xi_{x y}\right\|^{2}=\left(\nabla \xi_{x y}, \nabla(\varphi-\chi)\right), \quad \chi \in \mathscr{Q}, \\
& \left\|\xi_{x y}\right\|^{2} \leqq C\left\|\nabla \xi_{x y}\right\| \inf _{\chi \in \mathscr{2}}\|\nabla(\varphi-\chi)\| .
\end{aligned}
$$

The function $\xi_{x y}$ can be expanded in a double cosine series:

Thus,

$$
\xi_{x y}=\sum_{p, q=1}^{\infty} c_{p q} \cos \pi p x \cos \pi q y .
$$

$$
\varphi=\frac{1}{\pi^{2}} \sum_{\rho, q=1}^{\infty} \frac{c_{p q}}{p^{2}+q^{2}} \cos \pi p x \cos \pi q y .
$$

It then follows by approximating each product of cosines in $\mathscr{Q}$ that

$$
\inf _{\chi \in 2} \ddot{i} \vee(\varphi-\chi) \| i \leqq C h \ddot{\|} \xi_{x y} \ddot{\|}
$$

and the theorem has been proved.

Denote by $P$ the restriction of the projection $T$ to the subclass of $H^{1}(I)$ consisting of functions having zero average value. Let $\mathscr{P}=P \otimes P$.

We wish to obtain a better $H^{1}$ estimate of $v=\mathscr{P} z_{x y}-Z_{x y}$ than would follow from (6) and theorem 1. We deduce from (4) that

$$
(\nabla v, \nabla w)=\left(\nabla\left(\mathscr{P} z_{x y}-z_{x y}\right), \nabla w\right)=\tau_{x}+\tau_{y}, \quad w \in \mathscr{Q} .
$$

Using the definition of $P$ and integration by parts, we see that, for $w \in \mathscr{Q}$,

$$
\begin{aligned}
\tau_{x}= & \left(\left((I \otimes P)(P \otimes I) z_{x y}-z_{x y}\right)_{x}, w_{x}\right) \\
= & \left(I \otimes(P-I) z_{x x y}, w_{x}\right) \\
= & -\left(I \otimes(P-I) z_{x x x y}, w\right) \\
& +\left.\int_{0}^{1} I \otimes(P-I) z_{x x y}(., y) w(., y)\right|_{0} ^{1} d y .
\end{aligned}
$$


Note that $z$ has the representation

$$
\begin{aligned}
z(x, y)= & \int_{0}^{y} \int_{0}^{x}(x-\alpha)(y-\beta) u(\alpha, \beta) d \alpha d \beta \\
& -x \int_{0}^{y} \int_{0}^{1}(1-\alpha)(y-\beta) u(\alpha, \beta) d \alpha d \beta \\
& -y \int_{0}^{1} \int_{0}^{x}(x-\alpha)(1-\beta) u(\alpha, \beta) d \alpha d \beta \\
& +x y \int_{0}^{1} \int_{0}^{1}(1-\alpha)(1-\beta) u(\alpha, \beta) d \alpha d \beta
\end{aligned}
$$

One can easily verify from (9) that the boundary terms in (8) are zero since $z_{x x y}(0, y)=0$ and $z_{x x y}(1, y)=0$. We also observe that

$$
\int_{0}^{1} z_{x x x y} d y=z_{x x x}(x, 1)-z_{x x x}(x, 0)=0 \text {, }
$$

since $z$ vanishes on the boundary. Similarly, $\int_{0}^{1} z_{y y y x} d x=0$. Thus, we see that

where

$$
\|v\|_{1} \leqq C\|\psi\|_{-1}
$$

$$
\psi=I \otimes(I-P)\left(z_{x x x y}\right)+(I-P) \otimes I\left(z_{x y y y}\right) .
$$

It follows that

$$
\begin{aligned}
\|\psi\|_{-1} \leqq & \left(\int_{0}^{1}\left\|I \otimes(I-P) \frac{\partial^{4} z}{\partial x^{3} \partial y}(x, .)\right\|_{H^{-1}(I)}^{2} d x\right)^{1 / 2} \\
& +\left(\int_{0}^{1}\left\|(I-P) \otimes I \frac{\partial^{4} z}{\partial x \partial y^{3}}(., y)\right\|_{H^{-1}(I)}^{2} d y\right)^{1 / 2} .
\end{aligned}
$$

It is easy to show that

$$
\|(I-P) f\|_{H^{-1}(I)} \leqq C\left\|_{\mid} f^{(s)}\right\|_{L^{2}(I)} h^{s+1},
$$

provided that

$$
\int_{0}^{1} f d x=0
$$

by using the auxiliary problem

$$
\begin{gathered}
-\varphi^{\prime \prime}=g-\int_{0}^{1} g d x, \quad x \in 1, \\
\varphi^{\prime}(0)=\varphi^{\prime}(1)=0,
\end{gathered}
$$

vol. $11, \mathrm{n}^{\circ} 1,1977$ 
where $g \in H^{1}(I)$. Thus,

for $0 \leqq s \leqq r+1$.

$$
\|\psi\|_{-1} \leqq C\left\{\left\|\frac{\partial^{s+1} u}{\partial x^{s} \partial y}\right\|+\left\|\frac{\partial^{s+1} u}{\partial x \partial y^{s}}\right\|\right\} h^{s+2}
$$

THEOREM $2:$ Let $u$ be the solution to (1) with $L=\Delta$, and let $U \in M$ satisfy (2). $L e t \hat{U}$ be the $L_{2}$ projection of $u$ into $\mathscr{M}$. Then,

for $0 \leqq s \leqq r+1$.

$$
\|U-\hat{U}\| \leqq C\left\{\left\|\frac{\partial^{s+1} u}{\partial x^{s} \partial y}\right\|+\left\|\frac{\partial^{s+1} u}{\partial x \partial y^{s}}\right\|\right\} h^{s+1}
$$

Proof : Since $\hat{U}$ satisfies

$$
(\hat{U}-u, v)=0, \quad v \in \mathscr{M},
$$

one can easily verify that

$$
\hat{U}=\left(\mathscr{P} z_{x y}\right)_{x y} .
$$

Thus, (13) follows from (10), (12), and the quasi-uniformity hypothesis on the partition $\delta$.

COROLLARY : The error $U-u$ satisfies the following bounds:

$$
\begin{gathered}
\|U-u\| \leqq C\|u\|_{s} h^{s}, \quad 1 \leqq s \leqq r+1, \\
\|U-u\|_{L_{\infty}(\Omega)} \leqq C\left\{\|u\|_{W_{\infty}^{\infty}(\Omega)}+\left\|\frac{\partial^{s+1} u}{\partial x^{s} \partial y}\right\|+\left\|\frac{\partial^{s+1} u}{\partial x \partial y^{s}}\right\|\right\} h^{s} . \\
0 \leqq s \leqq r+1 .
\end{gathered}
$$

Proof: The $L_{2}(\Omega)$-estimate is a trivial consequence of (13). To obtain the $L_{\infty}(\Omega)$-estimate, note first that (13) and the quasi-uniformity of $\delta$ imply that, for $0 \leqq s \leqq r+1$,

$$
\|U-\hat{U}\|_{L \infty(\Omega)} \leqq\|v\|_{W_{\infty}^{2}(\Omega)} \leqq C\left\{\left\|\frac{\partial^{s+1} u}{\partial x^{s} \partial y}\right\|+\left\|\frac{\partial^{s+1} u}{\partial x \partial y^{s}}\right\|\right\} h^{s} .
$$

It follows from inequality (28) of [2] or from [1] that

$$
\|u-\hat{U}\|_{L_{\infty}(\Omega)} \leqq C\|u\|_{W_{\infty}^{s}(\Omega)} h^{s}, \quad 0 \leqq s \leqq r+1 .
$$

We now wish to consider the adjoint local $H^{-1}$ Galerkin procedure for $L=\Delta$. As noted earlier, there exists a unique $W \in \mathscr{N}$ satisfying

$$
(\Delta W, v)=(f, v), \quad v \in \mathscr{M} .
$$

THEOREM 3: Let $u$ be the solution to (1) with $L=\Delta$ and assume that $u_{x y} \in H^{s}(\Omega), 1 \leqq s \leqq r+2$. Let $W \in \mathscr{N}$ be defined by (14). Then,

$$
\left\|(W-u)_{x y}\right\|+h\left\|(W-u)_{x y}\right\|_{1} \leqq C\left\|u_{x y}\right\|_{s} h^{s} \text {. }
$$


Proof: Just as in (4),

$$
\left(\nabla(W-u)_{x y}, \nabla w_{x y}\right)=0, \quad w \in \mathscr{N}
$$

Since $w_{x y}$ represents an arbitrary element of $\mathscr{Q}$, the theorem follows from the analysis of (4) given in the proof of theorem 1 .

Next, we shall derive an $H^{1}(\Omega)$-estimate of the error $W-u$. Note that

$$
\begin{aligned}
\|\nabla(W-u)\|^{2} & =-(\Delta(W-u), W-u) \\
& =-(\Delta(W-u), W-u-\chi), \quad \chi \in \mathscr{M} .
\end{aligned}
$$

We choose $\chi \in \mathscr{M}$ as the local $H^{-1}$ Galerkin approximation to $W-u$; i. e.,

$$
(W-u-\chi, \Delta \varphi)=0, \quad \varphi \in \mathcal{N} .
$$

By the corollary to theorem 2,

$$
\|W-u-\chi\| \leqq C j \mid W-u \|_{1} h .
$$

From (15) and (16), we see that

$$
\|\nabla(W-u)\|^{2}=-(W-u-\chi, \Delta(W-u-\mu)), \quad \mu \in \mathcal{N} .
$$

Hence,

$$
\begin{aligned}
\|\nabla(W-u)\|^{2} & \leqq C h\|W-u\|_{1} \inf _{\mu \in \mathscr{N}}\|u-\mu\|_{2} \\
& \leqq C h^{s+1}\|W-u\|_{1}\|u\|_{s+2}, \quad 0 \leqq s \leqq r+1 .
\end{aligned}
$$

Since the boundary values of $u$ were imposed strongly on the elements of $\mathscr{N}$, the $L_{2}(\Omega)$-norm of the $\nabla(W-u)$ is equivalent to the $H^{1}(\Omega)$-norm of $W-u$; thus,

$$
\|W-u\|_{1} \leqq C\|u\|_{s+2} h^{s+1}, \quad 0 \leqq s \leqq r+1 .
$$

As a result of the quasi-uniformity of $\delta$, it follows easily that

$$
\|W-u\|_{2} \leqq C\|u\|_{s+2} h^{s}, \quad 0 \leqq s \leqq r+1 .
$$

Now, we shall seek an estimate of the error in $L_{2}(\Omega)$. Consider

$$
\begin{aligned}
& \Delta \varphi=W-u \quad \text { on } \Omega \text {, } \\
& \varphi=0 \quad \text { on } \partial \Omega \text {. }
\end{aligned}
$$

Then,

$$
\begin{aligned}
\|W-u\|^{2} & =(W-u, \Delta \varphi) \\
& =(\varphi, \Delta(W-u)) \\
& =\left(\varphi-\varphi^{*} . \Delta(W-u)\right), \quad \varphi^{*} \in \mathscr{M} .
\end{aligned}
$$

vol. $11, n^{\circ} 1,1977$ 
Thus, choosing an appropriate $\varphi^{*}$, we obtain the inequality

therefore,

$$
\begin{aligned}
\|W-u\|^{2} & \leqq C\|\varphi\|_{2} h^{2}\|\Delta(W-u)\| \\
& \leqq C\|W-u\|\|\Delta(W-u)\| h^{2} ;
\end{aligned}
$$

$$
\|W-u\| \leqq C\|u\|_{s+2} h^{s+2}, \quad 0 \leqq s \leqq r+1 .
$$

Summarizing the above results, we have proved the following theorem.

THEOREM 4: Let $u$ be the solution to (1) with $L=\Delta$ and assume that $u \in H^{s}(\Omega)$, $2 \leqq s \leqq r+3$. Then, if $W$ is defined by (14),

$$
\|W-u\|_{q} \leqq C\|u\|_{s} h^{s-q}, \quad .0 \leqq q \leqq 2 .
$$

If $k \geqq 0$, then the range on $q$ in theorem 4 can be extended to $0 \leqq q \leqq \min (k+3, s)$ by repeated use of quasi-uniformity to obtain the analogue of (17) in $H^{k+3}(\Omega)$.

\section{THE GENERAL CASE}

Let $U \in \mathscr{M}$ be determined as the solution of (2), and introduce an auxiliary function $U_{1} \in \mathscr{M}$ as the solution of

$$
\left(U_{1}-u, \Delta v\right)=0, \quad v \in \mathscr{N} .
$$

Let $\xi=U-U_{1}$, and let $\psi$ be given by the Dirichlet problem

Then, if $\psi^{*} \in \mathscr{N}$,

$$
\begin{array}{rll}
L^{*} \psi=\xi & \text { on } & \Omega, \\
\psi=0 & \text { on } & \partial \Omega .
\end{array}
$$

$$
\begin{aligned}
\|\xi\|^{2} & =\left(\xi, L^{*} \psi\right) \\
& =\left(\xi, L^{*}\left(\psi-\psi^{*}\right)\right)+\left(\xi, L^{*} \psi^{*}\right) \\
& =\left(\xi, L^{*}\left(\psi-\psi^{*}\right)\right)+\left(\eta, L^{*} \psi^{*}\right),
\end{aligned}
$$

where $\eta=u-U_{1}$. We choose $\psi^{*} \in M$ to satisfy

$$
\left(\Delta\left(\psi-\psi^{*}\right), v\right)=0, \quad v \in \mathscr{M}
$$

Thus, with $\tilde{b}$ and $\tilde{c}$ indicating the lower order coefficients of $L^{*}$,

$$
\begin{aligned}
\|\xi\|^{2}= & \left(a \xi, \Delta\left(\psi-\psi^{*}\right)\right)+\left(\xi, \tilde{b} \cdot \nabla\left(\psi-\psi^{*}\right)\right) \\
& +\left(\xi, \tilde{c}\left(\psi-\psi^{*}\right)\right)+\left(\eta, L^{*} \psi^{*}\right) \\
= & \left(a \xi-\chi, \Delta\left(\psi-\psi^{*}\right)\right)+\left(\xi, \tilde{b} \cdot \nabla\left(\psi-\psi^{*}\right)\right) \\
& +\left(\xi, \tilde{c}\left(\psi-\psi^{*}\right)\right)+\left(\eta, L^{*} \psi^{*}\right), \quad \chi \in \mathscr{M} .
\end{aligned}
$$


It is well-known that, since $a \in C^{1}(\bar{\Omega})$,

$$
\inf _{x \in \mathscr{M}}\|a \xi-\chi\| \leqq C\|\xi\| h .
$$

Replacing $u$ by $\psi$ and $W$ by $\psi^{*}$ in theorem 4 , we observe that

$$
\left\|\psi-\psi^{*}\right\|_{q} \leqq C\|\psi\|_{2} h^{2-q}, \quad 0 \leqq q \leqq 2 .
$$

Since $\|\psi\|_{2} \leqq C\|\xi\|$.

$$
\|\xi\|^{2} \leqq C\left\{h\|\xi\|^{2}+\|\eta\|\|\xi\|\right\} .
$$

Hence, for $h$ sufficiently small,

$$
\|\xi\| \leqq C\|\eta\| \text {. }
$$

Consequently, we have the following theorem.

THEOREM 5: There exists $h_{0}=h_{0}(L)>0$ such that a unique solution $U \in \mathscr{M}$ of (2) exists for $h \leqq h_{0}$; moreover, if $1 \leqq s \leqq r+1$ and if $u \in H^{s}(\Omega)$ is the solution of (1), then

$$
\|U-u\| \leqq C\|u\|_{s} h^{s}
$$

We shall now consider error estimates for the adjoint local $\mathrm{H}^{-1}$ Galerkin procedure. Note that the ellipticity of $L$ implies a Garding inequality of the form

$$
C_{0}\|\varphi\|_{1}^{2} \leqq-(L \varphi, \varphi)+C_{1}\|\varphi\|^{2}
$$

for $\varphi \in H^{2}(\Omega)$ such that $\varphi=0$ on $\partial \Omega$, where $C_{0}$ is some positive constant. Since (1) and (3) imply that $(L(W-u), \psi)=0$ for $\psi \in \mathscr{M}$,

$$
C_{0}\|W-u\|_{1}^{2}-C_{1}\|W-u\|^{2} \leqq-(L(W-u), W-u-\psi), \quad \psi \in \mathscr{M} .
$$

For $h$ sufficiently small, theorem 5 when applied to the operator $L^{*}$ instead of $L$ implies the existence of $\psi \in \mathscr{M}$ such that

$$
(L v, W-u-\psi)=0, \quad v \in \mathcal{N},
$$

and

$$
\|W-u-\psi\| \leqq C\|W-u\|_{1} h
$$

Thus, for any $\theta \in \mathscr{N}$ :

$$
\begin{aligned}
C_{0}\|W-u\|_{1}^{2}-C_{1}\|W-u\|^{2} & \leqq-(L(\theta-u), W-u-\dot{\psi}) \\
& \leqq C\|u-\theta\|_{2}\|W-u\|_{1} h .
\end{aligned}
$$

By noting that $\|W-u\|^{2} \leqq\|W-u\|_{1}\|W-u\|$, we see that

$$
\|W-u\|_{1} \leqq C\left(\|u\|_{s+2} h^{s+1}+\|W-u\|\right), \quad 0 \leqq s \leqq r+1 .
$$

vol. 11. $\mathrm{n}^{\circ} 1,1977$ 
Again by the quasi-uniformity of $\delta$,

$$
\|W-u\|_{2} \leqq C\left(\|u\|_{s+2} h^{s}+h^{-1}\|W-u\|\right), \quad 0 \leqq s \leqq r+1 .
$$

In order to obtain an $L_{2}(\Omega)$-estimate, we now consider the auxiliary Dirichlet problem given by

$$
\begin{aligned}
L^{*} \varphi & =W-u & & \text { on } \\
\varphi & =0 & \text { on } & \partial \Omega .
\end{aligned}
$$

Then,

$$
\begin{aligned}
\|W-u\|^{2}= & \left(W-u, L^{*} \varphi\right)=(L(W-u), \varphi) \\
& =\left(L(W-u), \varphi-\varphi^{*}\right), \quad \varphi^{*} \in \mathscr{M} .
\end{aligned}
$$

Thus, choosing an appropriate $\varphi^{*}$, we obtain the inequality

and

$$
\begin{aligned}
\|W-u\|^{2} & \leqq C\|W-u\|_{2}\|\varphi\|_{2} h^{2} \\
& \leqq C\|W-u\|_{2}\|W-u\| h^{2},
\end{aligned}
$$

$$
\begin{aligned}
\|W-u\| & \leqq C\|W-u\|_{2} h^{2} \\
& \leqq C\left(\|u\|_{s+2} h^{s+2}+\|W-u\| h\right), \quad 0 \leqq s \leqq r+1 .
\end{aligned}
$$

Hence, we have proved the following theorem.

THEOREM 6: There exists $h_{0}=h_{0}(L)>0$ such that there exists a unique solution $W \in \mathscr{N}$ of (3), and if $2 \leqq s \leqq r+3$ and if the solution $u$ of (1) belongs to $H^{s}(\Omega)$, then

$$
\|W-u\|_{\tilde{q}} \leqq C\left\|_{\|} u\right\|_{\bar{s}} h^{s-q}, \quad 0 \leqq q \leqq 2
$$

The range on $q$ can be extended just as for theorem 4 .

\section{REFERENCES}

1. J. Descloux, On Finite Element Matrices, S.I.A.M. J. Numer. Anal., Vol. 9, 1972, pp. $260-265$.

2. J. Douglas, Jr., T. Dupont and L. WAHLBIN, The stability in $L^{q}$ of the $L^{2}$-projection into finite element function spaces, Numer. Math., Vol. 23, 1975, pp. 193-197.

3. J.-L. Lions and E. MAgenes, Non-Homogeneous Boundary Value Problems and Applications, Vol. I, Springer-Verlag, New York, 1972. 